\title{
A Fisiologia em Educação Física e Esporte
}

\section{Cláudia Lúcia de Moraes FORJ AZ Valmor TRICOLI*}

*Escola de Educação Física e Esporte Universidade de São Paulo.

\section{Resumo}

A Fisiologia do Exercício, área de conhecimento derivada da Fisiologia, é caracterizada pelo estudo dos efeitos agudos e crônicos do exercício físico sobre as estruturas e as funções dos sistemas do corpo humano. Ela pode ser considerada uma das disciplinas mais tradicionais relacionadas à prática acadêmica e profissional da Educação Física e do Esporte em função da grande herança biológica destas áreas. Neste artigo, serão abordadas às linhas de pesquisa nessa área, a integração do conhecimento científico com a prática profissional e as perspectivas futuras da Fisiologia do Exercício.

Unitermos: Fisiologia do exercício; Fisiologia do esforço; Pesquisa; Profissão; Adaptação.

\section{Introdução}

O termo fisiologia vem do grego "physis" = natureza, função ou funcionamento e "logos" = palavra ou estudo. Assim, a Fisiologia caracteriza-se como o ramo da Biologia que estuda as múltiplas funçôes mecânicas, físicas e bioquímicas dos seres vivos. Ela se utiliza dos conceitos da física e da química para explicar como ocorrem as funçôes vitais dos diferentes organismos e suas adaptaçóes frente aos estímulos do meio ambiente.

Nesse contexto, a Fisiologia do Exercício (também chamada de Fisiologia do Esforço ou da Atividade Física) é uma área do conhecimento derivada da disciplina-mãe Fisiologia, que estuda como as funções orgânicas respondem e se adaptam ao estresse imposto pelo exercício físico (Joyer \& SAltin, 2008; WiLmORE \& Costill, 2010). Em outras palavras, a Fisiologia do Exercício estuda os efeitos agudos e crônicos do exercício físico sobre a estrutura e a função dos diversos sistemas orgânicos. Em complemento, a Fisiologia do Exercício investiga também a interação entre os diferentes efeitos do exercício físico e a influência dos estressores ambientais (Pate \& Durstine, 2004).

Entende-se por efeitos agudos, chamados de "respostas", as alterações decorrentes da execução de uma sessão de exercício. Essas respostas são subdivididas em respostas observadas durante o exercício (também chamadas de per exercício) e respostas observadas após o exercício (também chamadas de subagudas ou pós-exercício). As últimas podem ainda ser divididas em respostas imediatas, que ocorrem nas primeiras uma ou duas horas após o exercício, e tardias, que são observadas ao longo de 24 horas pós-exercício. Quanto aos efeitos crônicos, denominados "adaptaçóes", eles correspondem às alteraçóes estruturais e funcionais decorrentes de um período prolongado de treinamento físico regular (Nóbrega, 2005). Para melhor esclarecimento, podemos tomar como exemplo o interesse da área da Fisiologia do Exercício em investigar os efeitos da atividade física sobre a frequência cardíaca. Alguns pesquisadores poderiam estar interessados em saber se a frequência cardíaca se altera durante a execução de um exercício - efeitos agudos per exercício. Porém, outros poderiam estar interessados se, após a finalização do exercício, a frequência cardíaca retorna aos valores pré-exercício, caracterizando o estudo dos efeitos agudos pós-exercício. Outros ainda poderiam ter como objetivo saber se, após um período de treinamento de algumas semanas, a frequência cardíaca sofre alguma modificação efeito crônico. Em todas essas situações, a influência de outros fatores poderia também ser investigada, por exemplo, se esta resposta é a mesma em diferentes populaçôes, qual o efeito de diferentes tipos de exercício ou treinamento físico, ou ainda qual a influência da temperatura ambiental. Os mecanismos envolvidos nessas respostas também interessam aos pesquisadores e, portanto, também podem ser investigados. 
É interessante observar, entretanto, que pesquisas utilizando o exercício físico e avaliando as respostas fisiológicas são realizadas também por pesquisadores da fisiologia básica, sendo importante discriminar as diferenças dessas pesquisas para as pesquisas específicas, de caráter mais aplicado, em Fisiologia do Exercício. Para a fisiologia básica, o exercício é empregado como um estressor ao organismo, assim como poderia ser utilizado um estresse térmico, psicológico ou qualquer outro. O importante é colocar o organismo numa situação de instabilidade e verificar suas respostas. Fica claro que o exercício físico é utilizado como um meio de investigação científica. Por outro lado, para os pesquisadores da Fisiologia do Exercício, os conhecimentos da fisiologia básica são utilizados para explicar as respostas humanas ao exercício. O exercício passa a ser o ponto principal da análise, ele é o objeto de estudo propriamente dito, é a finalidade da pesquisa.

\section{Histórico}

As origens da Fisiologia do Exercício se confundem com os primórdios da Medicina e da prescrição da atividade física com fins terapêuticos no tratamento de doenças e manutenção das boas condições de saúde. Porém, somente no final do século 19 é que a Fisiologia do Exercício começou a surgir como uma área de interesse acadêmico-científico. O primeiro livro específico da área foi publicado em 1889 pelo pesquisador francês Fernand LaGrange intitulado "Physiology of Bodily Exercise" (WILmoRE \& Costill, 2010). É interessante destacar que a contribuição européia para a evolução da Fisiologia do Exercício prosseguiu nos anos seguintes. O dinamarquês August Krogh (1920), o britânico Archibald V. Hill (1922) e o alemão Otto Meyerhof (1922) receberam o Prêmio Nobel por suas pesquisas na fisiologia da musculatura esquelética e do metabolismo energético. Nos anos 30, os escandinavos Erik Hohwu-Christensen, Erling Asmussen e Marius Nielsen avançaram o conhecimento sobre as propriedades mecânicas do músculo esquelético e o controle da temperatura corporal em exercício. Christensen foi o mentor de Per-Olof Astrand, o qual obteve grande destaque nos anos 50-60 com investigaçōes relacionadas à aptidão física, saúde e resistência aeróbia. Ambos, Christensen e Astrand, foram mentores do sueco Bengt Saltin, que juntamente com Jonas Bergstrom, no final dos anos 60 ,
Alguns autores (PATE \& Durstine, 2004; WiLLMORE \& COSTILl, 2010) subdividem a Fisiologia do Exercício e conceituam a Fisiologia do Esporte como uma área do conhecimento que aplica os conceitos da Fisiologia do Exercício na elaboração e organização de meios, métodos e programas de treinamento voltados, especificamente, para o aumento do desempenho físico-esportivo de atletas.

Da mesma forma, outra subdivisão existente é a Fisiologia do Exercício Clínica, que aplica os conceitos da Fisiologia do Exercício na elaboração de programas voltados para manutenção da saúde, através da prevenção, tratamento e controle das doenças pelo exercício físico (EHRAN, GORDON, VISICH \& Keteyian, 2009; Pate \& Durstine, 2004).

Neste manuscrito, a terminologia Fisiologia do Exercício será utilizada para representar a aplicação dos conhecimentos fisiológicos às situações da Educação Física e do Esporte.

impulsionaram a aplicação da biópsia para o estudo da estrutura e da bioquímica muscular. Esta técnica permitiu aos fisiologistas do exercício compreender melhor o metabolismo energético e o efeito do tipo de fibra muscular no desempenho físico dos atletas.

Por 20 anos (1927-1947), o "Harvard Fatigue Laboratory" foi o ponto focal da história da Fisiologia do Exercício nos Estados Unidos da América. Neste laboratório, o Prof. Dr. David Bruce Dill, seu coordenador de pesquisa, conduziu estudos nas áreas de metabolismo energético, meio ambiente (efeitos do frio e da altitude), envelhecimento, nutrição e aptidão física e saúde (Powers \& Howley, 1994). Os anos 50 viram os nomes de Dudley Sargent e Thomas Cureton ganharem destaque. O livro clássico do Prof. Cureton "Physical Fitness of Champion Athletes", publicado em 1951, estimulou o interesse de diversos fisiologistas do exercício em investigar o perfil fisiológico de atletas (WiLmore, 2003). A partir dos anos 60, a Fisiologia do Exercício se estabeleceu como área de investigação científica com a presença de pesquisadores como William McArdle, Frank Katch, David Costill, Jack Wilmore, entre outros (DEVRIES, 2000).

No Brasil, a Fisiologia do Exercício teve início nos anos 70 com o Prof. Dr. Maurício Leal Rocha, profissional da área médica. Na década de 70 , todos os alunos que ingressavam na Universidade do Brasil 
(atual Universidade Federal do Rio de Janeiro UFRJ) passavam pelo Laboratório de Fisiologia do Exercício (LABOFISE), coordenado pelo Prof. Dr. Maurício, para medições antropométricas. A meta do professor era obter o perfil antropométrico e fisiológico dos alunos que ingressavam na Universidade. Esta iniciativa desencadeou alguns anos depois o Projeto Brasil. Este projeto propunha o deslocamento das avaliações para cidades do interior do país em busca do perfil de aptidão física do homem brasileiro. $\mathrm{O}$ Prof. Dr. Maurício esteve também envolvido com as primeiras atividades de ergometria, reabilitação e medicina do esporte no Brasil.

\section{Linhas gerais de investigação}

As investigações em Fisiologia do Exercício são bastante diversas e levam em conta diferentes aspectos relacionados aos efeitos do exercício. Considerando-se que esse efeitos sobre as diferentes funções orgânicas dependem das características do executante, as pesquisas na área da Fisiologia do Exercício têm sido desenvolvidas com diferentes populações: crianças, adolescentes, jovens, adultos, idosos, indivíduos saudáveis, portadores de doenças, sujeitos sedentários, condicionados e atletas de diferentes níveis e modalidades esportivas. Além disso, alguns estudos são conduzidos com animais de experimentação, como ratos, camundongos, coelhos, porcos, cachorros e gatos.

Classicamente, as pesquisas em Fisiologia do Exercício visam observar os efeitos do exercício em sistemas orgânicos específicos, tendo como os principais temas de investigação o metabolismo energético, os sistemas cardiorrespiratório, neuromuscular, imunológico e endócrino (Plowman \& SMith, 2009). Entretanto, durante o exercício físico ocorrem alterações simultâneas nas funções de todos estes sistemas, de modo que a tendência de investigação atual e, principalmente futura, na área da Fisiologia do Exercício é a elaboração de pesquisas que tragam uma visão mais abrangente e integrada dos efeitos do exercício no organismo como um todo.

Considerando-se as linhas de investigação da Fisiologia do Exercício, algumas subáreas podem ser identificadas. Os estudos iniciais se concentravamse, principalmente, em aspectos da aptidão física e desempenho esportivo, enquanto que, mais recentemente, um grande volume de conhecimento passou a ser produzido sobre aspectos relacionados à saúde. Dessa forma, é possível subdividir a Fisiologia do
A história da Fisiologia do Exercício na Escola de Educação Física e Esporte da Universidade de São Paulo (EEFEUSP) começou com o primeiro laboratório de pesquisa da Escola denominado CIPEF - Centro Integrado de Pesquisa em Educação Física. Este centro de pesquisa foi criado por um docente de formação na área de medicina, o Prof. Dr. Mário de Carvalho Pini e foi coordenado pela Profa. Dra. Maria Augusta Peduti Dal'Molin Kiss. O CIPEF desenvolveu as primeiras pesquisas na área da Fisiologia do Exercício e foi responsável por formar alguns dos pesquisadores que se tornaram líderes nesta especialidade em nosso país (TANI, 1999).

Exercício em aspectos relacionados ao desempenho esportivo e aspectos relacionados à aplicação clínica (Ehran et al., 2009; PATE \& Durstine, 2004). As pesquisas relacionadas à primeira área envolvem a avaliação de atletas, procedimentos de treinamento, desenvolvimento de capacidades motoras, respostas agudas e adaptações ao processo treinamento, efeitos do meio ambiente, entre outros. $\mathrm{Na}$ área da Fisiologia do Exercício voltada à saúde, os estudos abordam a prevenção, tratamento e controle de doenças relacionadas à hipocinesia (i.e. oriundas da baixa participação em atividades físicas), em especial, as doenças crônico-degenerativas.

Para explicar os efeitos agudos e crônicos do exercício sobre as funções orgânicas em sua plenitude, a Fisiologia do Exercício precisa não só descrever as respostas observadas em decorrência da execução do exercício e do treinamento físico, mas também precisa explicar os mecanismos envolvidos e a aplicabilidade desses achados em condiçõos reais. Para cumprir esse papel, a Fisiologia do Exercício envolve pesquisas de cunho básico e aplicado. No contexto básico, os estudos investigam os mecanismos; enquanto que as pesquisas aplicadas testam diferentes características do exercício físico em diferentes populações e avaliam os efeitos dessas diferenças nas respostas agudas e crônicas das funçôes orgânicas (THOMAS \& NELSON, 2005).

A pesquisa aplicada envolve ainda dois níveis de investigação: o clínico ou de laboratório e o prático ou de aplicação em campo. No nível clínico, a hipótese de estudo é testada em condições bem controladas, o que aumenta a validade interna do estudo, ou seja, a chance daquele resultado realmente ser consequência da intervenção realizada. 
Por outro lado, no nível da aplicação em campo, a investigação da hipótese ocorre em condiçōes reais de execução, com menor controle das variáveis que podem interferir nos resultados, o que aumenta sua validade externa, ou seja, a chance do resultado ser extrapolado para situações semelhantes.

Para facilitar a compreensão, vamos retornar ao exemplo sobre o que acontece com a frequência cardíaca durante a execução do exercício. Nesse contexto, a pesquisa clínica ou de laboratório poderia medir a resposta da frequência cardíaca durante um determinado exercício (por exemplo, 20 minutos pedalando no cicloergômetro com 100 watts), tentando controlar todos os outros fatores que poderiam afetála como, por exemplo, a temperatura e hora do dia. Essa investigação poderia descobrir que a frequência cardíaca aumenta $50 \%$ durante a execução desse exercício nestas condições. A pesquisa aplicada de campo, por outro lado, usaria esse mesmo exercício numa situação real, por exemplo, em pessoas que se exercitam num clube, sem controlar a temperatura e hora do dia e, dessa forma, verificaria se, realmente, a frequência cardíaca aumenta durante a execução. Ainda no mesmo contexto, a pesquisa básica tentaria explicar quais são os mecanismos responsáveis pelo aumento da frequência cardíaca durante o exercício. Eventualmente, a investigação de mecanismos necessita se aprofundar tanto, que por falta de tecnologia

\section{Linhas de pesquisa}

Diversas definiçôes de linha de pesquisa podem ser observadas. Segundo Borges-ANDRADE (2003), a linha de pesquisa pode se entendida como um traço imaginário que: a) determina o rumo ou o que será investigado; b) delimita as fronteiras do campo específico do conhecimento que será abordado; c) oferece orientação teórica aos que farão a pesquisa; e d) estabelece os procedimentos adequados.

Segundo a Coordenação de Aperfeiçoamento de Pessoal de Nível Superior (CAPES), agência do governo federal que determina os rumos da pós-graduação brasileira, linha de pesquisa define um domínio ou núcleo temático da atividade de pesquisa de um Programa de Pós-Graduação, que envolve o desenvolvimento sistemático de trabalhos com objetos ou metodologias comuns. Já para o Conselho Nacional de Desenvolvimento Científico e Tecnológico (CNPq), agência do Ministério da Ciência e Tecnologia destinada ao fomento da ou por questóes éticas, ela precisa ser conduzida em animais e, nesse caso, um modelo animal adequado precisaria ser escolhido.

A transição do conhecimento da pesquisa básica para a clínica de laboratório e aplicada de campo é conhecida como pesquisa translacional. No cenário científico é altamente desejado que este modelo seja utilizado para que o fenômeno investigado seja explicado de forma mais abrangente e completa (LIPPI, 2011). Cabe ressaltar, no entanto, que a sequência de evolução do conhecimento entre esses tipos de pesquisa não precisa seguir um sentido único. Em outras palavras, é possível que a pesquisa básica crie uma hipótese que seja testada pela clínica de laboratório e depois de campo, assim, como às vezes, a pesquisa de campo cria hipóteses para serem testadas pela pesquisa de laboratório e cujos mecanismos precisam ser investigados pela pesquisa básica.

Para finalizar, é importante ressaltar que para que a ciência não perca o foco da realidade, é fundamental que as pesquisas nos diferentes níveis (básica e aplicada) embasem suas buscas em questôes pertinentes e relacionadas à atuação da Educação Física e do Esporte. Em outras palavras, é desejável que o campo profissional traga os problemas que enfrenta para serem investigados, e que as hipóteses sejam montadas em função desses problemas e que sejam testadas de forma integrada nos diferentes níveis de pesquisa.

pesquisa científica e tecnológica e à formação de recursos humanos para a pesquisa no Brasil, linhas de pesquisa representam temas aglutinadores de estudos técnico-científicos, que se fundamentam em tradição investigativa, de onde se originam projetos cujos resultados guardam relação entre si.

Analisando-se as definiçōes anteriores, fica claro que uma linha de pesquisa deve ser abrangente o bastante para aglutinar pesquisas com temas e métodos comuns, mas também deve ser delimitada o bastante para que seu rumo e campo específico de investigação não se percam. Essa dificuldade de abrangência faz com que as linhas de pesquisa sejam muitas vezes definidas de forma imprecisa.

Infelizmente, essa mesma falta de precisão observada na definição das linhas de pesquisa da Educação Física e do Esporte, também pode ser verificada na área da Fisiologia do Exercício. Em levantamento no diretório dos grupos de pesquisa do CNPq (http://

10 • Rev. bras. Educ. Fís. Esporte, São Paulo, v.25, p.7-13, dez. 2011 N. esp. 
www.cnpq.br/gpesq/apresentacao.htm), usando-se como palavra chave de busca, Fisiologia do Exercício, observam-se desde linhas de pesquisa muito abrangentes, como "Fisiologia do Exercício", "Impacto do exercício físico aeróbico e resistido, agudo e crônico sobre os sistemas fisiológicos de indivíduos saudáveis e indivíduos doentes" ou "Atividade física, aptidão física e saúde", que quase se caracterizam como áreas do conhecimento; até linhas muito delimitadas, como "Estudo das técnicas de avaliação física e antropométrica: análise crítica, interpretação e aplicação prática" ou "Estudo da variabilidade da frequência cardíaca em pacientes com fibromialgia - efeito do treinamento resistido", que quase se configuram como um projeto de pesquisa.

Apesar dessa grande imprecisão na definição das linhas e sua abrangência, é possível observar que as linhas de pesquisas em Fisiologia do Exercício se situam, em sua maioria, em investigações voltadas para um conhecimento mais aplicado. Estudos sobre as alteraçóes fisiológicas geradas por um tipo específico de exercício físico, a avaliação de parâmetros fisiológicos durante o exercício, os efeitos do exercício físico relacionados à saúde ou ao desempenho físico-esportivo são temas frequentemente encontrados. Além disso, algumas linhas de pesquisa definem já em seu título a população alvo de estudo (crianças, adultos, idosos, sujeitos saudáveis, portadores de doenças, atletas e animais). O tipo de exercício ou de treinamento também tem sido incluído no tema de algumas linhas de pesquisa, como treinamento de força, treinamento aeróbio, ciclismo, caminhada, entre outros. Algumas vezes, o sistema orgânico que será investigado também é citado, ou seja, adaptações cardiovasculares, respiratórias, endócrinas, imunológicas, etc.

Para exemplificar, são apresentadas, a seguir, algumas das linhas mais comuns de pesquisa dentro da Fisiologia do Exercício: 1) efeitos do exercício/treinamento físico sobre os sistemas muscular e ósseo; 2) respostas cardiorrespiratórias ao exercício físico; 3) efeitos agudos e crônicos do exercício no sistema cardiovascular; 4) diagnóstico da aptidão aeróbia; 5) exercício físico e estresse oxidativo; 6) efeitos agudos e crônicos do exercício físico no sistema imune; 7) efeitos agudos e crônicos do exercício no sistema endócrino; 8) exercício físico e envelhecimento; e 9) exercício físico e doenças crônico-degenerativas.

\section{Integração com o campo profissional}

O conhecimento acadêmico-científico é primordial para uma formação profissional adequada e, principalmente, para o oferecimento de um serviço de qualidade para a sociedade. Assim, a relação entre a produção do conhecimento científico em Fisiologia do Exercício e o campo de atuação profissional da Educação Física e do Esporte deve ser considerada como uma etapa importante no processo de formação do futuro profissional.

Cabe lembrar que os conhecimentos oriundos $\mathrm{da}$ Fisiologia do Exercício podem não ser aplicados de forma direta na prática profissional, mas oferecem suporte para o conhecimento aplicado produzido por áreas como o Treinamento Esportivo e a Atividade Física para a saúde. Entender como o corpo humano funciona e como ele reage e se adapta frente aos estímulos oferecidos pela prática de exercícios físicos permite a busca por soluçóes mais adequadas para a melhoria da saúde e do rendimento físico-esportivo.

Segundo Plowan e Smith (2009), o conhecimento oferecido pela Fisiologia do Exercício permite ao aluno de graduação: a) compreender como o exercício físico modifica o funcionamento fisiológico básico do organismo do ser humano em curto e longo prazo, conhecendo os mecanismos responsáveis por essas mudanças (o conhecimento da resposta normal permite reconhecer uma resposta anormal e adaptar-se a ela); b) proporcionar programas de educação física e esporte que estimulem o praticante tanto física quanto intelectualmente (os praticantes precisam compreender como o exercício físico pode beneficiálos, porque são avaliados e como os resultados dessa avaliação podem ser utilizados); c) ser capaz de aplicar os resultados da pesquisa científica de forma a maximizar a saúde, a reabilitação e/ou o desempenho atlético em uma ampla variedade de populaçôes; e d) ser capaz de responder com embasamento científico às questões e alegaçóes da propaganda, bem como de reconhecer e reagir aos mitos e concepçóes errôneas sobre o exercício e a prática esportiva.

Portanto, o conhecimento cientificamente orientado permitirá ao profissional elaborar intervençōes baseadas nas respostas fisiológicas previsíveis em curto, médio e longo prazos para obter os objetivos almejados. Além disso, ele será capaz de avaliar essas respostas e, se necessário, modificar a estratégia de intervenção. 


\section{Perpectivas futuras}

A compreensão de como o corpo humano responde fisiologicamente a cargas agudas e crônicas de exercício físico evoluiu muito nos últimos 50 anos. Os grandes avanços nos recursos tecnológicos para pesquisa e a melhoria na qualidade e na quantidade de recursos humanos envolvidos com a produção do conhecimento na área da Fisiologia do Exercício foram fatores primordiais nesta evolução.

Atualmente, a Fisiologia do Exercício constitui uma área de pesquisa bastante abrangente que tem recebido influência de diferentes áreas e de novos métodos de investigação científica. Isto tem permitido uma maior velocidade na descoberta de mecanismos, inclusive em nível celular e molecular, o que era anteriormente impossível. Estudos que antes levavam dias para a coleta e tratamento dos dados hoje podem ser feitos em questão de horas. Até mesmo a confecção de manuscritos tornou-se mais veloz.
Com certeza, os próximos 20 anos irão apresentar uma evolução marcante e uma exploração ainda maior, em particular, em nível microscópico, molecular e genético dos eventos associados à adaptação do corpo humano ao exercício físico e à influência do meio ambiente.

Evidentemente, a transferência dos conhecimentos para a prática não é tão simples e depende de um bom entendimento da área. A simples produção de conhecimento não garante a melhoria na prática profissional. Muitos dos pesquisadores atuantes na Fisiologia do Exercício não possuem sua formação de origem na Educação Física ou no Esporte. Ao mesmo tempo em que isto é interessante para a expansão do conhecimento e da interdisciplinaridade, cuidado deve ser tomado para que este progresso no sistema de investigação não distancie a pesquisa em Fisiologia do Exercício dos problemas e necessidades acadêmicas e profissionais da Educação Física e do Esporte.

\begin{abstract}
The Physiology in Physical Education and Sport

Exercise Physiology is a sub discipline from the area of Physiology. It is characterized by the investigation of the acute and chronic effects of physical exercise on the structures and functions of the human body. Exercise Physiology can be considered one of the most traditional academic and professional sub disciplines of the Physical Education and Sport because of the biological heritage of these fields. In this paper, we are going to discuss the lines of investigation, the relationship between the scientific knowledge and professional practice, and the future perspectives for the Exercise Physiology.
\end{abstract}

UnITERMS: Exercise physiology; Research; Profession; Adaptation.

\title{
Referências
}

BORGES-ANDRADE, J.E. Em busca do conceito de linha de pesquisa. Revista de Administração Contemporânea, Curitiba, v.7, n.2, p.157-70, 2003.

deVRIES, H. History of exercise science. In: HOUSH, T.J.; HOUSH, D.J. (Eds.). Introduction to exercise science. Boston: Allyn \& Bacon, 2000. p.12-35.

EHRMAN, J.E.; GORDON, P.M.; VISICH, P.S.; KETEYIAN, S.K. Clinical exercise physiology. Champaign: Human Kinetics, 2009.

JOYER, M.J.; SALTIN, B. Exercise physiology and human performance: systems biology before systems biology. Journal of Physiology, Bethesda, v.589, n.1, p.9, 2008.

LIPP, G. Translational research in sport science. British Journal of Sports Medicine, London, v.45, n.3, p.167, 2011.

PATE, R.P.; DURSTINE, J.L. Exercise physiology and its role in the clinical sports medicine. Southern Medical Journal, Birmingham, v.97, n.9, p.881-5, 2004.

12 • Rev. bras. Educ. Fís. Esporte, São Paulo, v.25, p.7-13, dez. 2011 N. esp. 
PLOWMAN, S.A.; SMITH, D.L. Fisiologia do exercício para saúde, aptidão e desempenho. Rio de Janeiro: Guanabara Koogan, 2009.

POWERS, S.K.; HOWLEY, E.T. Exercise physiology: theory and applications to fitness and performance. Dubuque: Brown \& Benchmark, 1994.

NOBREGA, A.C.L. The subacute effects of exercise: concept, characteristics, and clinical implications. Exercise Sport Science Reviews, New York, v.33, n.2, p.84-7, 2005.

TANI, G. Atividade de pesquisa na Escola de Educação Física e Esporte da Universidade de São Paulo: passado, presente e futuro. Revista Paulista de Educação Física, São Paulo, v.13, p.20-35, 1999. Número especial.

THOMAS, J.R.; NELSON, J.K. Research methods in physical activity. Champaign: Human Kinetics, 2005.

WILMORE, J.H. Applied exercise physiology: a personal perspective of the past, present, and future. Exercise and Sport Sciences Reviews, New York, v.31, n.4, p.159-60, 2003.

WILMORE, J.H.: COSTILL, D. L. Fisiologia do esporte e do exercício. Barueri: Manole, 2010.

\author{
ENDEREÇO \\ Valmor Tricoli
}

Escola de Educação Física e Esporte - USP

Av. Prof. Mello Moraes, 65

05508-030 - São Paulo - SP - BRASIL

e-mail: vtricoli@usp.br 